REVISTA DE DERECHO UNED, núm. 3, 2008

\title{
REFLEXIONES SOBRE LA NOCIÓN DE IUSTITIA EN LA TRADICIÓN JURÍDICA EUROPEA
}

\author{
LEO PEPPE \\ *traducido por Jesus Gómez Garzás
}

Resumen: Nella tradizione giuridica europea la formulazione più famosa della nozione di giustizia è espressa con il sintagma cuique suum. Prendendo avvio dalla notizia che all'ingresso del campo di concentramento nazista di Buchenwald era posta la scritta Jedem das Seine ('A ciascuno il suo'), viene ripercorsa la storia della formula e delle sue utilizzazioni nei suoi momenti fondamentali: nel diritto romano (con una sua evoluzione, ma sempre all'interno del concreto ordinamento giuridico e sociale); agli inizi del pensiero cristiano (Ambrogio, Agostino); nel medio evo (Tommaso d'Aquino, il diritto canonico); nell'età moderna (Spinoza, Leibniz, Kant); fino all'età contemporanea, quando ormai la formula da una parte è venuta assumendo contenuti giusnaturalistici estranei alle origini romane, dall'altra viene accusata di poter esprimere qualsiasi contenuto (Kelsen). Si è altresì evidenziata l'esistenza della forma particolare unicuique suum, che ricorre quasi esclusivamente nella tradizione e nei documenti della Chiesa Cattolica, dal medio evo ad oggi, con un uso nel quale appare accentuato il valore attivo della formula: la iustitia è actus iustitiae.

Palabras clave: Tradición juridica - justicia ? cuique suum ? iustitia ? a cada uno lo suyo ? derecho romano ? Iglesia ? derecho natural Buchenwald

Abstract: In the European legal tradition the expression cuique suum is the most famous formulation of the idea of ?justice?. The starting point of the article is the consideration that at the entrance of the Nazi lager Buchenwald was placed the maxim Jedem das Seine (?To each his own?). The A. studies the history of the formula and its uses 
in key moments: the Roman Law (in its own evolution, but always concerning the concrete legal and social system); at the beginning of the Christian Thought (Ambrose, Augustin); in the Middle Age (Thomas Aquinas, the Canon Law); in the Modern Age (Spinoza, Leibniz, Kant); in the Contemporary Age, when the wording on one hand has jusnaturalistic contents unrelated to the Roman origins, on the other it is accused of having undetermined meaning (Kelsen). The A. points out that the particular shape unicuique suum is used nearly exclusively in the tradition and in documents of Roman Catholic Church, from Middle Age today, with a use in which appears been accentuated the active value of the formula: iustitia is actus iustitiae.

Palabras clave: Justice ? cuique suum ? iustitia ? his own ? Roman Law? Church ? Natural Law- Buchenwald

1. El objeto de este trabajo son algunas fórmulas atribuidas al derecho romano y añadidos entorno a la noción de iustitia: fórmulas que examino de nuevo en el contexto de la historia jurídica europea y desde una particular perspectiva, la de la visión histórica e historiográfica del nazismo ${ }^{1}$.

2. Me dirijo a una pregunta angustiosa retomada aún en años muy recientes: ¿Cómo ha sido posible, en la cultura europea, llegar del romano cuique suum al lema jedem das Seine colocado en la entrada del campo de concentración nazi de Buchenwald? En el 2005, el histórico holandés del derecho intermedio van den Bergh ha determinado la respuesta en la moderna concepción racionalista y positivista del derecho, contraponiendo a esta concepción una lectura de las fuentes romanas en términos de valores filtrados por la jurisprudencia.

Más claramente, otros estudiosos, como el filósofo del derecho alemán Klenner, han rebatido la convicción ya expresada por Kelsen (y posteriormente por Topitsch) sobre el sintagma cuique suum: éste, al igual que el jedem das Seine, por sí mismo no explica un contenido, sino que de vez en cuando lo recibe del concreto ordenamiento; por

1 Per quanto si dirà nel testo e per la letteratura di riferimento si rinvia in primo luogo a L. PEPPE, 'Jedem das Seine', "(uni)cuique suum", 'a ciascuno il suo', in AA. VV., Tradizione romanistica e Costituzione (diretto da L. LABRUNA, a cura di M.P. BACCARI-C. CASCIONE), Napoli, 2006, t. II (Collana „Cinquanta anni della Corte costituzionale della Repubblica italianas), p. 1707 ss. 
tanto, esta expresión debe ser considerada una fórmula vacía. En el mismo sentido también se manifiesta, en 2001, Jutta Limbach, entonces Presidente de la Corte Constitucional de la República Federal Alemana.

De esta opinión también es Gustavo Zagrebelsky, ex presidente de la Corte Constitucional italiana, quien en 2004 escribía: «tomemos la más famosa y comprensiva entre las fórmulas de la justicia, el uni cuique suum tribuere, el «a cada uno lo suyo» de los jurisconsultos romanos, o su reformulación "trata a los iguales de modo igual, y a los diferentes de modo diferente". ... Fórmulas como estas pueden ser acogidas por cualquiera (...). Los campos de exterminio, por ejemplo, están en regla con esta máxima de la justicia. El modo de bienvenida al campo de Buchenwald (...) era, precisamente, jedem das Seine,...».

En estas frases está resumido, de la mejor y más sintética manera, la relación entre las nociones de justicia y las fórmulas que constituyen el objeto de mi intervención.

3. La proximidad entre el principio de derecho romano y el lema nazi resulta, a primera vista, fuertemente sugestiva; éstos parecen estar superpuestos: en algunos autores resulta explícita la hipótesis de una relación de descendencia de uno del otro; en otros autores, además, el lema de Buchenwald es utilizado como momento de verificación teórica de la interpretación dada a la formulación construida por los jurisconsultos romanos. Esta utilización de principios jurídicos romanos puede parecer "continuista», es decir, pretenden afirmar una ininterrupida continuidad desde Roma hasta hoy; o bien, puede parecer descontextualizada, esto es, fuera de los contextos históricos específicos. De cualquier modo, una utilización similar puede provocar, en el estudioso del derecho romano, una reacción de cautela acerca de la legitimidad de la superposición y, por tanto, de la representación dada; en mi caso, esta reacción de cautela deriva de algunas vicisitudes; por ejemplo cuando he verificado que el brocardo Societas delinquere non potest (a menudo atribuido al derecho romano) en realidad tenía, ciertamente, una historia mucho más reciente.

Seguramente no es éste el supuesto de cuique suum, que tiene una historia, al menos, de igual complejidad, sino ciertamente mucho más documentada y segura en sus ascendencias romanas. No obstante, existe un punto de estrecho contacto con la problemática y el tiempo que aquí nos interesa; además societas delinquere non potest ha sido empleado en el proceso de Nuremberg (un proceso fuertemente inspirado en el derecho natural y, por ello, implicada la noción de justicia) por el defensor de la Gestapo, que lo ha recordado 
-como un principio de derecho romano- a fin de excluir la responsabilidad de la organización en cuanto tal.

A veces, además, el uso de una categoria romana puede resultar, a primera vista, fascinante y útil para explicar una realidad casi contemporánea, para, a continuación, resultar fundado sobre una interpretación de las fuentes romanas inaceptables por parte de un iusromanista. Es el supuesto de la representación de los perseguidos por el nazismo hecha por el filósofo italiano Agamben en 1995, en los términos romanos de homo sacer, excluido por la comunidad: una interpretación fascinante pero infundada.

Así, viene a emerger el ejemplo de un uso difuso del derecho romano por parte de no iusromanistas. Los iusromanistas ciertamente no pueden desconocer la legitimidad de estas operaciones, a menudo conducidas con un buen conocimiento de los instrumentos técnicos necesarios: personalmente he podido constatar como esto resulta bastante útil en las múltiples reuniones científicas organizadas por ARISTEC.

Los riesgos aludidos en el uso del derecho romano (y en general de la cultura clásica) están bien presentes, incluso en los niveles más altos, por ejemplo el del constituyente europeo: hasta casi el momento de la firma en Roma, el 29 de Octubre de 2004, del Tratado que adopta una Constitución para Europa, se había puesto, en el inicio del Tratado mismo, una pésima cita de Tucídides (Tucídides II, 37): esta cita fue eliminada del texto provisional sólo unos meses antes, el 18 de junio de 2004.

En conclusión, esta cautela, a mi entender indispensable en el uso de los materiales «romanos" y clásicos, debe ser encuadrada en el más amplio y complejo contexto de las relaciones entre la historia del derecho (entendida también como parte de la "cultura clásica) y el derecho positivo, contexto en el que, además, no resulta secundario el papel de la historia del derecho en la formación del jurista, siempre más necesariamente europeo. Y esto resulta, tanto más necesario, cuando la formación del jurista mira a la justicia, a sus parámetros.

4. Pero antepuesto todo esto, no se puede negar que, en el dramático contexto del nazismo, los principios del derecho romano parecen emerger, de diverso modo, como un punto de referencia ideal, en el bien y en el mal. Por lo demás, verdaderamente en la experiencia del área alemana, el derecho romano ha conocido repetidamente (y cíclicamente) los dos extremos, de la adopción plena (de la Recepción a la Pandectística) al rechazo más preciso (desde Lutero al nazismo). 
Detengámonos ahora en el Jedem das Seine, el lema colocado en la entrada del campo de Buchenwald; el pensamiento se dirige rápidamente al análogo Arbeit macht frei de Auschwitz I. ¿Cuál es el origen de estos lemas? En síntexis puede decirse que el sistema nazi de los campos de concentración se injerta sobre una realidad ya existente en los Strafgefangenenllager, pero modificándola inmediatamente en 1933 por orden de Himmler, a partir de Cachau, donde nace el conocido Arbeit macht frei, también usado sucesivamente para otros campos diferentes. Las razones de la utilización del lema en Dachau no son conocidas, pero el lema parece tener sus orígenes, de cualquier modo, en matrices ideológicas recientes, casi contemporáneas.

Sólo una parte de los campos de concentración resulta haber recibido un lema para su ingreso, seis, quizá siete. En cuanto a Buchenwald, junto a Weimar, este campo constituye una excepción respecto a la difusión del lema Arbeit macht frei.

La historia del campo se inicia en Julio de 1937, con bastante probabilidad, en febrero/marzo de 1938 se realiza el lema Jedem das Seine.

Faltan datos ciertos idóneos para explicar la elección de este lema para éste (y sólo éste) campo, en aquel momento. Se han hecho muchas conjeturas, pero ciertamente, a diferencia del lema Arbeit macht frei, el lema Jedem das Seine tiene, en la historia del lenguaje y de la cultura alemana, una vicisitud plurisecular; compleja y bastante articulada en la sociedad misma; ciertamente —en los estratos más cultos de la sociedad - corresponde al cuique suum romano (obviamente bien presente en aquella cultura); se puede además recordar que «suum cuique» era el lema del máximo orden prusiano, «Der Hohe Orden von Schwarzen Adler», fundado en 1701 por Federico I de Prusia; o bien se recuerda la Cantata BWV 163, Nur jedem das Seine, compuesta por J.S. Bach en 1715, sobre el texto de Salomo Franck, quien había estudiado derecho en Jena.

De todos modos, ante la falta de certeza, prefiero concluir con la constación de que en 1938, cuando el lema es adoptado por Buchenwald, esto sucede en el interior de una cultura nazi que había hecho propio lo que era un viejo dicho alemán, acentuándo el posible valor discriminatorio y convirtiéndolo en un «slogan» para una política social y jurídica que sobreentendía, no una mera atribución de derechos o al menos de expectativas, sino la exclusión de algunos de los derechos, de aquellos derechos que ahora correspondían sólo a los puros arios alemanes, sujetos únicamente a la voluntad del Führer. A 
los demás le corresponde la total exclusión, después Vernichtung, aniquilamiento, como ciudadanos, trabajadores, creyentes.

Todo esto acerca de la vertiente perteneciente a la edad moderna, del lema Jedem das Seine. Pero ¿qué se debe decir por cuanto respecta a la fórmula romana unicuique suum tribuere recordada por Zagrebelsky y por él unida al lema alemán? Subrayo en seguida: unicuique, non cuique, como estamos habituados a leer.

5. Esta diferencia lexical podría ser irrelevante, pero es en las pequeñas diferencias en las que a veces se puede encontrar la huella de algo más importante. El discurso no puede empezar sino por las fuentes romanas. No me detengo aquí en el origen del cuique suum en la filosofía griega, en la concepción de la justicia distributiva, lo que aquí se muestran son los textos romanos: la mayor parte de estos textos son conocidísimos, algunos de ellos se encuentran en la compilación justinianea, en posición inicial y, por ello, privilegiada.

Las Institutiones de Justiniano comienzan así:

Inst. 1.1.1: Iustitia est constans et perpetua voluntas ius suum cuique tribuens. Iuris prudentia est divinarum atque humanarum rerum notitia, iusti atque iniusti scientia.

Y poco después, se lee en Inst. 1.1.3: Iuris praecepta sunt haec: honeste vivere, alterum non laedere, suum cuique tribuere.

$\mathrm{Al}$ inicio del Digesto, Ulpiano hace derivar ius de iustitia, recuerda la definición de Celso del ius como ars boni et aequi, reivindica la cualidad de sacerdotes de los juristas, en cuanto que cultivan la justicia, «iustitiam colimus». Poco después define iustitia, iuris praecepta, iuris prudentia: D. 1.1.10: Iustitia est constans et perpetua voluntas ius suum cuique tribuendi. 1. Iuris praecepta sunt haec: honeste vivere, alterum non laedere, suum cuique tribuere. 2. Iuris prudentia est divinarum atque humanarum rerum notitia, iusti atque iniusti scientia.

De este modo hemos recordado los testimonios del Corpus Iuris; si omitimos una cita de Catón el Censor, a mi entender ajena a nuestro discurso, puede decirse que la verdadera historia de la fórmula cuique suum se inicia en Roma con la Rhetorica ad Herennium (que durante muchos siglos será considerada obra juvenil de Cicerón) y el De inventione del propio Cicerón: dos obras datadas entorno a los años 8483 a.C.

Rhetorica ad Herennium. 3.2.3: Rectum est, quod cum virtute et officio fit. It dividitur in prudentiam, iustitiam, fortitudinem, modes- 
tiam. ... Iustitia est aequitas ius uni cuique retribuens pro dignitate cuiusque. De este texto puedo destacar cuatro datos:

a) éste es el único texto de la «fórmula» en el que, antes del Corpus Iuris, aparecía ius;

b) sólo en este texto (y en Cic. De inv. 2.53.160, que se tratará en breve) se utiliza —en relación con la fórmula que nos interesa- la dignitas, circunstancia a mi entender bastante importante;

c) además, éste es el único texto donde se emplea unicuique, en lugar de cuique, para designar el destinatario de la atribución, pero con una especificidad única que quizá tenga algún significado y, por tanto, pueda contribuir a explicar la pareja semántica unicuique/cuique. La dignitas es cuiusque, de cada uno, se refiere a un sujeto no determinado: a él se atribuye el ius, en razón y proporción de su dignitas, y en este punto se indica el destinatario con un reforzamiento de su designación de individualidad, con unicuique: exclusivo a él, en su específico derecho (y, en efecto, hay ius) individual que le corresponde (retribuens), presupuesto genéricamente en la dignitas cuiusque. Quizá esta definición de iustitia es la más auténticamente romana;

d) en estos términos existe correspondencia entre el derecho que debe ser atribuido y la dignitas de cada uno; pero en la sociedad y en el derecho romano la dignitas es tan importante que puede llegar arrebatar el quantum de derecho que le habría correspondido (o no correspondido), como sucede en el famoso caso del joven estudiante de Digesto 5.1.18.1 (donde se lee, in fine: ...nonne augetur utilitas per dignitatem?).

Someto, por tanto, a su atención CIC. De inv. 2.53.160: iustitia est habitus animi, communi utilitate conservata, suam cuique tribuens dignitatem. Si recordamos lo que acabamos de exponer acerca de Rhet. Ad Her. 3.2.3, y se comparan los dos textos, se pueden determinar dos aspectos de la reflexión ciceroniana en el De Inventione: de una parte la commnunis utilitas se pone como un dato necesario a preservar; de otra parte, existe ciertamente una modalidad expresiva más breve, porque Cicerón no utiliza el término ius para indicar el objeto del tribuere valorado en relación a la dignitas; él resume sintéticamente tal objeto en la sua dignitas: esta última operación podrá parecer sólo una simplificación, pero, en realidad, se trata de algo mucho más complejo, con un resultado final quizá más riguroso desde el punto de vista filosófico: la dignitas no es más que el criterio de la atribución, se convierte en una palabra que resume la posición individual, también resulta una palabra aún profundamente romana 
y, por ello, evocadora de la complejidad de la atribución. El siguiente paso será la eliminación de la mención de la dignitas: aproximadamente treinta años después en el 83 a.C., en un tiempo totalmente diferente de la sociedad romana y de la vida de Cicerón, existen una serie de textos ciceronianos.

CIC. De leg. 1.6.19: Itaque arbitrantur prudentiam esse legem, cuius ea vis sit, ut recte facere iubeat, vetet delinquere, eamque rem illi Graeco putant nomine 'nómon' a suum cuique tribuendo appellatam, ego nostro a legendo;

CIC. De fin. 5.23.65: Quae animi affectio suum cuique tribuens ... iustitia dicitur;

CIC. De fin. 5.23.67: Atque haec coniunctio confusioque virtutum tamen a philosophis ratione quadam distinguitur. Nam cum ita copulatae conexaeque sint, ut omnes omnium participes sint nec alia ab alia possit separari, tamen proprium suum cuiusque munus est, ut fortitudo in laboribus periculisque cernatur, temperantia in praetermittendis voluptatibus, prudentia in dilectu bonorum et malorum, iustitia in suo cuique tribuendo. Quando igitur inest in omni virtute cura quaedam quasi foras spectans aliosque appetens atque complectens, existit illud, ut amici, ut fratres, ut propinqui, ut affines, ut cives, ut omnes denique - quoniam unam societatem hominum esse volumus - propter se expetendi sint. Atqui eorum nihil est eius generis, ut sit in fine atque extremo bonorum;

CIC. De nat. deorum 3.15.38: Nam iustitia, quae suum cuique distribuit, quid pertinet ad deos; hominum enim societas et communitas, ut vos dicitis, iustitiam procreavit;

CIC. De off. 1.5.15: Sed omne quod est honestum, id quattuor partium oritur ex aliqua: aut enim in perspicientia veri sollertiaque versatur aut in hominum societate tuenda tribuendoque suum cuique et rerum contractarum fide aut in animi ... magnitudine ... aut in omnium ... ordine et modo, ...;

CIC. De off. 1.7.21: Ex quo, quia suum cuiusque fit eorum quae natura fuerunt communia, quod cuique obtigit, id quisque teneat;

LACT. epit. 50 [55], 5-8, dal De rep.; CIC. de rep. 3.7.10: Plurimi quidem philosophorum, sed maxime Plato et Aristoteles, de iustitia multa dixerunt adserentes et extollentes eam summa laude virtutem, quod suum cuique tribuat, quod aequitatem in omnibus servet.

Aquí permanece sólo el suum, lo que es suyo, se podría decir basado en la ontología de modo genérico; posteriormente, más de dos 
siglos después, se da (en Digesto 1.1.10 pr.) la redefinición del objeto de la atribución de la iustitia por parte de Ulpiano: no más la sua dignitas, no más el suum, sino el ius suum.

Al final de esta reseña de textos, debe recordarse a Séneca y a los escritores cristianos, como Paolo, Agustín, Ambrosio. En conclusión, de esta sintética presentación de textos relevantes, debe formularse una advertencia: es importante subrayar que muy a menudo estos textos son citados por ediciones no recientes, o bien en los mismos textos han sido realizadas pequeñas modificaciones, o bien para conformar la citación, o bien para poder insertarla en un determinado contexto.

6. Pero detengámonos ahora en un dato lingüístico ya aludido, la existencia de dos versiones de la "fórmula» ya examinada: unicuique suum o cuique suum, esta última - hasta ahora- aparece de forma más frecuente. En realidad, todavía hoy, la versión unicuique suum es empleada de forma bien visible: de hecho aparece en la cabecera del Osservatore romano, el periódico oficial de la Santa Sede: a esas palabras se acompañan estas otras, non praevalebunt. ¿Por qué utiliza aquí unicuique y no cuique? Unusquisque no se emplea -en particular como unicuique - en ninguno de los textos y autores que, aquí, han sido tomados en consideración hasta ahora: en ellos se encuentra siempre y sólo cuique, con la única excepción de Rhet. Ad Her. 3.2.3, que es también el único texto en el que, en lugar de suum, emplea ius (pero no ius suum, el sintagma que, en cambio,se emplea en el Corpus iuris).

Entre las fuentes cristianas utilizables, en su opinión, Klenner cita a Pablo, Ad Gal. 6.5., donde, en efecto, emplea unusquisque, pero el contexto no resulta del todo homogéneo en relación con lo que aquí interesa; además resulta seguramente del todo pertinente SAN AGUSTIN. De civitate Dei, 19,21,1: Iustitia porro ea virtus est quae sua cuique distribuit. El contexto de este pasaje de San Agustín es aquél de una dura comparación con Cicerón, y resulta evidente la procedencia de esta definición de iustitia, de aquélla ya encontrada en un pasaje ciceroniano (De nat. Deorum 3,15,38: ...iustitia, quae suum cuique distribuit ...): aún emplea cuique.

7. Por tanto, resulta necesario profundizar en el discurso, comenzando desde L'Osservatore romano. Este periódico inicia su publicación el uno de Julio de 1861, los dos lemas son añadidos posteriormente en el número uno del año siguiente, donde se explica que non praevalebunt es una cita: Portae inferi non praevalebunt, esto es Matías 16.18, el texto evangélico sobre el que se edificó la Iglesia de 
Roma. En cuanto al unicuique suum, éste no viene fijado por una cita, sino que se explica descendiendo a la dimensión humana, aquélla de orden natural divino. Los dos lemas son unidos ("el nexo lógico y moral que los enlaza en un sólo concepto») en la función de baluarte de la Iglesia romana contra la revolución, en primer lugar la «revolución italiana»; recuerdo que había sido a continuación de la unificación de Italia bajo la monarquía de los Saboya.

Pero en realidad unicuique suum es una expresión que pertenece a la más profunda tradición cristiana y católica, en su construcción aristotélico-tomista en particular, pero no únicamente; en otras ocasiones ha sido empleada por el propio papa Juan Pablo II.

El punto de referencia no puede ser otro que Tomás de Aquino; en concreto, en la Summa Theologiae, es posible encontrar muchos pasajes en los que se emplea la terminología que aquí nos interesa, en concreto en las quaestiones II,II,57-60.

Pero ya en el año 1234, por tanto, antes de Santo Tomás, es posible leer una frase de extraordinario interés y de la máxima autoridad; esta frase se encuentra al inicio de la Bula «Rex pacificus», que emana los Decretalium Gregorii Papae IX Compilationis libri V: Ideoque lex proditur, ut appetitus noxius sub iuris regula limitetur, per quam genus humanum, ut honeste vivat, alterum non laedat, ius suum unicuique tribuat, informatur.

En realidad, es posible retroceder en el tiempo, en la tradición cristiana; de hecho unicuique se emplea constantemente en este contexto de «atribución de esto que es suum/ius suum» ya en un anónimo monasterio cisterciense de los inicios del siglo XII de la abadía de Santa María de la Ferrara en Vairano (en la provincia de Caserta), en Abelardo, en Gregorio VII, también en San Isidoro de Sevilla, en los albores del Medievo, se encuentra cuique.

8. Ya se puede ver en Santo Tomás, pero resulta evidente cómo, todavía hoy, los textos antiguos son leídos en la forma unicuique de los eclesiásticos. Particularmente interesante resulta cómo esto también sucede a propósito de un texto de San Ambrosio, cuya edición correcta es AMBROS. De off. 1.24.115: Quarum [virtutum] primo loco constituerunt prudentiam [...]; secundo iustitiam quae suum cuique tribuit, alienum non vindicat, utilitatem propriam neglegit ut communem aequitatem custodiat.

En síntexis conclusiva puede afirmarse que, en consecuencia, el único unicuique, de nuestro contexto, de edad romana, parece estar en Rhet. Ad Her. 3.2.3 (donde no obstante se emplea ius y no suum), 
por lo que parece plausible que nos encontremos ante una tradición cristiana relativamente tardía, ya formada -como se ha visto- en los siglos precedentes a Santo Tomás y que, en su obra, resulta definitivamente adquirida.

Probablemente no resulta forzado encontrar en esta formulación unicuique suum otra cosa más diversa respecto a la tradición (sea también con alguna incerteza) cuique suum de las escuelas civilistas: esto es un diverso valor semántico en el contexto de la tradición cristiana y después católica, ahora ya, como se podría deducir del uso tradicional en Melantone, en 1543. Puede, por tanto, decirse que si en cuique suum el suum es aquéllo que corresponde (= que otros, el ordenamiento, etc., dicen que corresponde), en unicuique quod suum est el suum es aquéllo que es ya suum, el derecho ni lo considera ni asume las defensas, la iustitia es actus iustitiae.

Cierro estas consideraciones lingüísticas con dos últimas observaciones: en primer lugar la dicción unicuique resulta, por tanto, bastante difundida, a partir de alguna edición de la Glosa ordinaria; pero resulta también atestiguada, por ejemplo, más veces en el breve trascurso del tiempo, en la Dalmacia medieval.

En segundo lugar querría recordar, en relación a esta plausible tradición "eclesiástico /romana», un texto que me parece bastante interesante por su originalidad. Se trata de un procedimiento jurisdiccional (un investimentum) del Senado Romano del 1162 d.C., en realidad, un gran tribunal, que se puede insertar en el cuadro de la primera fase de la historia del senado romano medieval; este procedimiento se inicia con la siguiente fórmula: Nos senatores pro iustitia cuique tribuenda a reverendo atque magnifico populo Romano in Capitolio costitui. ¿De qué se trata? El Senado romano, en este caso, es llamado a decidir la controversia acerca de la propiedad de la pequeña iglesia de San Nicolas a los pies de la Columna Trajana entre el presbitero de la iglesia, Angelo, y la abadesa de San Ciriaco en Via Lata. El Senado atribuye la iglesia y la columna a la abadesa, pero debe ser respetado el decoro público de la ciudad, salvo honore publico urbis: de hecho en el mismo tiempo la columna debe ser conservada tal y como está in eterno. Muerte y confiscación de todos los bienes a cualquiera que viole esta regla. Resulta interesante señalar que, de este modo, son tutelados el interés privado y la utilidad pública: se podría decir que el Senado de la Roma medieval decide conforme al ya citado Cicerón: De inventione 2.53.160 (iustitia est habitus animi, communi utilitate conservata, suam cuique tribuens dignitatem ...) y con anticipador conocimiento de la impor- 
tancia hoy atribuida a los «bienes culturales» privados y a su conservación.

9. Hasta ahora, con lo que se ha dicho, sólo se ha aclarado algún aspecto de la historia del sintagma latino que constituye el objeto de mi intervención. Ahora quisiera intentar precisar mejor el significado de aquel sintagma: la afirmación de lo inadecuado de la «fórmula» es un dato recurrente en la teoría general del derecho, no de hoy: resulta suficiente recordar la critica kelseniana al suum cuique como tautológica reducción ( «inhaltsleer») del derecho de cada cual al ordenamiento jurídico positivo que constituye el presupuesto. Pero ya Spinoza, de modo ejemplar, decía que, verdaderamente porque en la naturaleza el derecho de cada uno es, en definitiva, función y expresión de su poder, puede decirse que el ius suum de cada uno no puede existir en el estado de natura, sino sólo en el estado civil: es la feroz similitud de los peces en el Tratado teológico-político: pisces summo naturali iure aqua potiuntur, et magni minores comedunt; los peces son señores del agua según el sumo derecho natural y los peces grandes se comen a los peces pequeños.

En definitiva, la fórmula, en sí considerada, se agota en el derecho positivo, cualquier derecho positivo. Alguien podría decir que nunca habría sido posible colocar cuique suum/jedem das Seine en la entrada de Buchenwald si, como canta Bach en la ya citada Cantata BWV 163, existe un Dios al que mirar, el hombre no está sólo con César: sino que las concretas vicisitudes de la historia no confortan esta afirmación. Al mismo tiempo, ¿es posible una alternativa laica, un punto de referencia laica que impida la redución del cuique suum y del derecho al derecho positivo? Es esto lo que buscan todos aquellos que aspiran a conectar normas y valores, también hoy. Sobre este vínculo, obviamente el discurso se termina con un último dato de interése: como se ha visto, la "fórmula" cuique suum, en las fuentes romanas se emplea, ya sea aisladamente, ya sea en el interior de un trío de reglas, acompañando al alterum non laedere y al honeste vivere. Las tres reglas pueden ser consideradas separadamente, pero también unitariamente, al menos con la enorme fuerza de un modelo traslaticio imprescindible, hasta poderse poner como los tres indispensables praecepta de un ius que no sería tal si uno de los tres faltara. Sobre esta posibilidad interpretativa, en cuanto se refiere a las fuentes romanas, se volverá en breve; tal posibilidad ciertamente ha sido seguida en época moderna. Se podrían dar muchos ejemplos, recuerdo sólo dos relevantes, Leibniz en la Nova Methodus y Kant en Die Metaphysik der Sitten, dos iusnaturalistas diferentes entre sí, ambos protagonistas de la trayectoria moderna de subjetivización del derecho y 
de la realidad. En particular, en la Rechtslehre, Kant recuerda los tres praecepta ulpianeos, pero los reformula y en ellos encuentra los deberes jurídicos, los Rechtspflichte; y se debe entrar en el estado civil porque sólo en éste, ésto es legalmente determinado, aquello que para cada uno es suyo; es «el poder judicial (que asigna a cada uno según la ley) en la persona del juez».

10. En este punto, para comprender el significado de estas utilizaciones modernas del lema cuique suum el discurso debe volver a las fuentes romanas: para leerlas no sólo en la clave del uso que ha sido hecho sucesivamente sino para intentar leer aquello que efectivamente decían, en su tiempo, al prescindir de las lecturas sucesivas.

Estos textos en realidad son netamente divisibles en dos grupos, según el tradicional criterio de distinción entre fuentes jurídicas y fuentes extrajurídicas: en este caso la distinción resulta particularmente importante y vital, por que el objeto del discurso no es un precepto específico, eficaz en su imperatividad y operatividad concreta, para lo cual la juridicidad sería in re ipsa, sino generales y genéricos conceptos de iustitia y de iuris praecepta, por los que la carga jurídica es menos directa, y la cualidad de las fuentes se vuelve, entonces, esencial.

10.1. A las fuentes jurídicas, evidentemente, pertenecen los ya citados Inst. 1.1.1., Inst. 1.1.3, D. 1.1.10, a los que generalmente se le asocia D.50.17.144pr., del jurista Paulo, como la siguiente pieza de una posible o hipotética "teoría general" romana: no todo lo que es consentido es honesto. D. 50.17.144 pr.: Non omne quod licet honestum est. En realidad esta conexión resulta sugestiva pero viene a conferir a la frase una cualidad programática que no tiene en el Digesto, ni mucho menos podía tener en su contexto originario. También, queriendo conferir mayor "aislamiento" a esta frase de Paulo respecto a la institución originaria, se encuentra el hecho de que la reflexión ulpianea, que hemos visto en D.1.1.10, se coloca en posición casi inicial en el Digesto justinianeo y constituye una regula, no sólo por el contexto, sino porque nace como regula, proveniendo desde el primero de los Regularum libri VII de Ulpiano: por la naturaleza misma de la regula, aquella reflexión se sustancia en reglas abstractas, principios jurídicos; no en el caso, en la terminología propia del Common Law, por tanto serán asumidas como «legal maxims» y, en consecuencia, recordadas al público en lugares emblemáticos, como es la sala de lecturas de la Harvard Law School.

Por cuanto se refiere a los iuris praecepta, ha habido quien ha considerado que Ulpiano resume «positivamente» los fundamentos de 
la convivencia civil, los «iuris praecepta»; por contra, algunos los reducen al rango de "Leerformeln", meras enunciaciones; en fin, una posición intermedia coloca sobre el plano de las enunciaciones de principios, ya sea el alterum non laedere, ya sea el honeste vivere, afirmando, además, la validez (en sentido jurídico) de la regla suum cuique propio para el papel del jurista en el sistema romano de producción del derecho.

10.2. A mi parecer, aceptando la icástica formulación de Villey, quien afirma que con estas expresiones el derecho romano no describe «des droits, mais des status». Y, si se acepta esta conclusión, este puede ser probablemente el punto de mayor diferencia entre el valor romano de la fórmula (la relación entre el quisque y el todo) respecto a aquélla moderna (la relación entre los singulares sujetos respecto al todo).

Desde esta perspectiva, el suum cuique romano no tiene contenido alguno de justicia en términos de valores o, mucho menos, de derechos humanos; pero, sobre todo, no tiene como punto de referencia el suum (un contenido merecedor de tutela) que corresponde a un individuo, en cuanto tal, abstracto portador de derechos (en la versión más completa: entre iguales): ni, por lo demás, la sociedad romana (su filosofía, su derecho) podía dis/tribuere el suum desde esta perspectiva estrechamente individualista, una revolución de la edad moderna y, antes todavía, se debe recordar frente a recientísimas polémicas italianas, del subjetivismo cristiano. Y, en efecto, ius y iustitia en los inicios del Digesto justinianeo no son distintos (con la significativa alteración en la etimología de ius, que se hace derivar de iustitia), pero son unificados en el unitario ámbito del bonum et aequum: el derecho deriva de la justicia, la justicia no es un mundo de valores sino un parámetro, un criterio, "cultivado" por los juristas. Lo que resulta opuesto al pensamiento del glossatore Piacentino, muerto en 1192, que corta la relación entre ius y iustitia: author iuris homo, iustitiae Deus; aquél Piacentino tenía bien presente la relación entre los juristas y el poder político.

11. Concluyo. Las reflexiones apenas desarrolladas muestran la complejidad y problemática de cualquier intento de reconstrucción de la historia del pensamiento romano sobre este tema y constituyen, en cierta medida, la perspectiva necesaria sobre la que leer la «vera philosophia» de los juristas romanos; pero con el conocimiento de que, en el mismo pensamiento romano, deben ser hallados dos diversos momentos: el primero, aquél del suum cuique de la sociedad de los señores, diverso por la dignitas, en época republicana; el se- 
gundo, aquel del suum cuique de los cives todos iguales, pero subiecti, esto es, súbditos, en época imperial.

En consecuencia, Ulpiano (y con él la compilación justinianea) podría colocarse en esta nueva concepción de la iustitia, para la cual es objeto el suum; pero Ulpiano es jurista y, para él, la definición de iustitia no sería tal si no contuviese la referencia al derecho, tanto más si, de una parte, ius deriva de iustitia - como Ulpiano mismo afirma en el primer libro de sus Institutiones - y, de la otra, ius puede ser enlazado a la iustitia representada como aequitas (otro término fundamental del ius civile), como ya aparece en Rhet. Ad Her.3.2.3. Se encuentran, entonces, los tres iuris praecepta, para los que se caracterizan tres ámbitos de acción humana, de los que sólo el tercero es suum cuique tribuere. No ius suum cuique, pero esto resulta obvio si se obvserva la finalidad para la que es construida la frase, indicar las tres reglas de oro del derecho: éstas, por definición, pueden tener por objeto únicamente una situación jurídica. Esta conclusión hace inútil la repetición de ius en ius suum, y atrae de forma plena a la esfera jurídica los otros dos preceptos; antes, en esta perspectiva, repetir ius habría empobrecido la fuerza jurídica de los otros praecepta.

En el pensamiento cristiano se puede hallar una reflexión similar a la ulpianea, pero diferente, no obstante, por muchos aspectos. Es el TEXTO de San Ambrosio (De off. 1.24.115): secundo iustitiam quae suum cuique tribuit, alienum non vindicat, utilitatem propriam neglegit ut communem aequitatem custodiat.

Los motivos de interés son más de uno, también la comparación con el De officiis ciceroniano muestra una simplificación problemática; y resultan evidentes, en particular el suum cuique se recurre por primera vez, el honeste vivere es sustituido por la centralidad de la communis aequitas, finalmente el alterum non laedere es, a su vez, sustituido por el alienum non vindicat. Particularmente interesante parece, también, este último punto ya que el principio del alterum non laedere -del que frecuentemente se afirma hoy su inconsistencia jurídica - viene representado en su concreta juridicidad.

En Ambrosio quizá ya existe una mayor tensión acerca de comportamientos concretos, tomando el camino hacia la iustitia como actus iustitiae en Tommaso (y en el completo pensamiento cristiano): Proprius actus iustitiae nihil est aliud quam reddere unicuique quod suum est.

En conclusión, la historia de los usos de cuique suum (y unicuique suum) podría ser la mejor prueba de que esta fórmula no tiene un 
constante y definido contenido concreto. Creo que se puede concluir que se trata de una regla destinada a operar de modo relativo (junto con otras reglas), y funcional respecto al ordenamiento en el que ésta es incluida.

En realidad, el cuique suum de la justicia distributiva romana al concreto civis ha conservado en algunos contextos de la edad moderna este significado originario, pero prevalentemente se ha convertido en aquéllo que corresponde a cada uno en razón de su individualidad de ser humano, por derecho natural humano o divino y en un determinado ordenamiento jurídico, con sus normas fundamentales (no matar, etc...): es, respecto a este segundo significado de la fórmula, lo que hace que el uso del lema en Buchenwald aparezca increible e insoportable. Pero quizá no les habría parecido insoportable a los Romanos, si recordamos, en el 70 d.C., el relato de Flavio Giuseppe, los fugitivos desde la Jerusalem asediada eran crucificados de quinientos en quinientos, y además en un sólo día: la vida de un enemigo no constituía ningún valor y no merecía respeto, ni siquiera en la muerte. 